\title{
Philosophiques
}

\section{Réflexion complice et réflexion purifiante chez Sartre et Heidegger}

\section{Antoine Hatzenberger}

Volume 25, numéro 1, printemps 1998

URI : https://id.erudit.org/iderudit/027472ar

DOI : https://doi.org/10.7202/027472ar

Aller au sommaire du numéro

Éditeur(s)

Société de philosophie du Québec

ISSN

0316-2923 (imprimé)

1492-1391 (numérique)

Découvrir la revue

Citer cet article

Hatzenberger, A. (1998). Réflexion complice et réflexion purifiante chez Sartre et Heidegger. Philosophiques, 25(1), 63-71. https://doi.org/10.7202/027472ar
Résumé de l'article

A plusieurs reprises, mais de manière assez allusive, Sartre, dans L'être et le néant, met en cause une perspective éthique inavouée à l'oeuvre dans Etre et temps. Ne peut-on, à partir de cette critique fragmentée de Heidegger, saisir les jeux de miroir qui existent entre ces deux oeuvres ? Ne doit-on pas interpréter ce mouvement de distanciation comme une tentative désespérée de différer le problème moral ? Une lecture conjointe de L'être et le néant et de Etre et temps centrée sur la distinction entre réflexion complice et réflexion pure ne permet-elle pas d'entrevoir les difficultés que pose à l'ontologie phénoménologique la question de l'éthique? 


\title{
RÉFIEXION COMPLICE FI RÉFIEXION PURIFIANIE CHEZ SARTRE G HEIDEGGER
}

\author{
PAR \\ ANTOINE HATZENBERGER
}

RÉSUMÉ : À plusieurs reprises, mais de manière assez allusive, Sartre, dans L'être et le néant, met en cause une perspective éthique inavouée à l'ouuvre dans Être et temps. Ne peut-on, à partir de cette critique fragmentée de Heidegger, saisir les jeux de miroir qui existent entre ces deux auvres? Ne doit-on pas interpréter ce mouvement de distanciation comme une tentative désespérée de différer le problème moral ? Une lecture conjointe de L'être et le néant et de Être et temps centrée sur la distinction entre réflexion complice et réflexion pure ne permet-elle pas d'entrevoir les difficultés que pose à l'ontologie phénoménologique la question de l'éthique?

ABSTRACT: In Being and Nothingness, Sartre on several occasions, but rather allusively, questions an inavowed ethical perspective at work in Being and Time. Is it then possible, from this fragmented critique of Heidegger, to capture the specularity which exists between these two works? Should this distancing be interpreted as a desperate attempt to differ the moral problem? How can a joint reading of Being and Nothingness and Being and Time focused on the distinction between réflexion complice and réflexion pure allow us to perceive the difficulties which the question of ethics creates for phenomenological ontology?

Le rapport de L'être et le néant à $\hat{E}$ tre et temps n'est pas simple. Prises en elles-mêmes, ces deux œuvres philosophiques majeures de notre siècle s'offrent à nous dans la succession chronologique. Mais, dans les nombreuses références à Heidegger, et surtout par les nombreuses critiques qu'il lui adresse, Sartre met en place un rapport complexe de type reflété-reflétant. Niant ce qui pourtant de bout en bout le sous-tend, $L$ 'être et le néant est, pourrait-on dire, par rapport à Être et temps comme le pour-soi par rapport à l'en-soi. Heidegger est là, mais toujours sur le mode de la négation. 
De la conscience, radicalement remise en question par Heidegger, Sartre fait un point de départ ${ }^{1}$. Il relativise l'importance insigne accordée à la mort dans $\hat{E}$ tre et temps ${ }^{2}$. Et, pour lui, le point de vue de Heidegger sur la question cruciale d'autrui n'échappe pas, en définitive, à l'idéalisme ${ }^{3}$. Sartre trouve même à redire contre le style du philosophe allemand caractérisé comme " manière brusque et un peu barbare de trancher les nœuds gordiens ${ }^{4}$ ". Enfin, et surtout, à plusieurs reprises, et de manière assez allusive, Sartre critique une certaine mauvaise foi de Heidegger qui aurait introduit subrepticement un point de vue moral dans Etre et temps, une éthique inavouée :

|... la description de Heidlegger laisse trop clairement paraître le souci de foncler ontologiquement une Éthique dont il prétend ne pas se préoccuper ${ }^{5}$.

[... les expressions "authentique "et " inauthentique "[que Heidegger] emploie [sont] douteuses et peu sincères à cause de leur contenu moral implicite ${ }^{6}$.

C'est donc par rapport à ce qu'on pourrait appeler le problème moral que le mouvement de reprise critique d'un système philosophique par l'autre pose les problèmes les plus délicats. L'interrogation sur ce problème moral est sollicitée par le paradoxe devant lequel nous place $L$ 'être et le néant : Sartre semble voir dans Être et temps ce que justement Heidegger affirme nettement n'y être pas. En effet, celui-ci a établi le cadre méthodologique de l'interprétation du phénomène du on en se tenant loin de toute perspective éthique :

[...] l'interprétation a une intention purement ontologique, elle est donc on ne peut plus éloignée d'une critique moralisante du Dasein quotidien?.

$[\ldots]$ l'interprétation ontologique existentielle ne se prononce $|\ldots|$ pas $[\ldots]$ ontiquement sur la " corruption de la nature humaine ", non parce que les moyens pour la prouver font défaut, mais parce que sa problématique se tient en deçà de tout énoncé sur la corruption ou la non-corruptions.

1. Sartre, Lêtre et le néant, Paris, Gallimard, 1943 : "il faut partir du cogito " (p. 112).

2. Heidlegger procède par un " tour de passe-passe "; sa pensée de la mort se meut dans " un cercle" (ibid., p. 591). "Ainsi, nous devons conclure, contre Heidegger, que loin que la mort soit ma possibilité propre, elle est un fait contingent qui, en tant (fue tel, m’échappe par principe et ressortit originellement à ma facticité. " (p. 603)

3. "Heidegger n'échappe pas à l'idéalisme. " "Il serait vain $[\ldots]$ de chercher dans Sein und Zeit le dépassement simultané de tout idéalisme et de tout réalisme. » (Ibid., p. 295)

4. Ibid, p. 290.

5. Ibid., p. 118.

6. Ibid., p. 588 .

7. Heidegger, Etre et temps, trad. de F. Vezin, Paris, Gallimard, 1986, p. 214 [167].

8. Ibid., §38, p. $227[179-180]$. 
On peut alors se demander si, dans ce dialogue qu'il instaure, dans cette relation critique qu'il met en place à l'encontre d'une de ses références constitutives, en fait, Sartre ne met pas en évidence certains points problématiques de son propre système. Tout au moins, ne met-il pas par là l'accent sur un certain inachèvement explicitement assumé par ailleurs - de ce système?

Pour essayer d'éclairer le sens de cette critique paradoxale', et de distinguer par là ce qu'elle indique en termes d'opposition et surtout de parallélisme entre $L$ être et le néant et k̂tre et temps, il faut saisir le problème moral là où il se pose, c’est-à-dire sur le lerrain de la distinction heideggerienne entre authenticité et inauthenticité ${ }^{10}$.

Pour le Dasein, l’inauthenticité est le mode d'être le plus courant, quotidien, car" "d'abord et le plus souvent, le Dasein ne fait qu'un avec le on ${ }^{11}$ ». C'est le mode d'être dans lequel le Dasein ne peut avoir qu'une compréhension impropre de lui-même, ne comprenant qu'improprement le temps, la mort... Mais ce qu'il faut souligner, c'est que l'inauthenticité n’est aucunement marquée d'un quelconque discrédit moral; dans le $\$ 9$, Heidegger pose que "l'impropriété du Dasein ne signifie [...] pas une sorte de " moindre " être ou un niveau d'être " dégradé "12 "par rapport au Dasein authentique. Dit autrement, "[l]e phénomène du dévalement [Verfallen] ne présente pas $[\ldots]$ quelque chose comme un " côté sombre "du Dasein, une qualité survenant ontiquement et pouvant servir à nuancer le tableau un peu trop riant de cet étant. Le dévalement révèle une structure ontologique tenant à l'essence du Dasein lui-même qui en accuse d'autant moins le côté noclurne qu'elle configure tous ses jours dans leur quotidienneté ${ }^{13}$ ".

Cette notion de Verfallen ne doit pas évoquer le sens moral moralisateur - de la chute ${ }^{14}$. Certes, a [l] e Dasein chute en lui-même à partir de lui-même, il plonge dans le vide et l'inanité de la quotidienneté impropre ", mais le rapport authenticitéinauthenticité est un rapport de simultanéité : le Dasein est tout à la fois authentique et inauthentique. Ciest Sartre qui semble introduire. une nuance morale dans cette distinction en faisant de ce rapport un

9. L'étude de cette critique paradoxale fait délibérément abstraction de toute stratégie de distinction. Stratégie clont l'éventualité est évoquée par Alain Renaut, dans Sartre, le dernier philosophe, Paris, Grasset, 1993, p. 59: "Dans ces réserves, il faut sans doute $[\ldots]$ faire la part des stratégies de clistinction ru'un auteur débutant (après tout, $L$ être et le néant est le premier ouvrage véritablement important cle Sartre en philosophie) estime si souvent avoir besoin de déployer, vis-à-vis de son trop proche inspirateur, afin d'affirmer sa propre pensée ".

10. E. Martineau traduit Eigentlichkeit et Uneigentlichkeit par authenticité et inauthenticité (Être et temps, Paris, Authentica, 1985) et $\mathrm{F}$. Vezin par propriété et impropriété.

11. Ibid., p. $214[167]$.

12. Ibid., p. $74[43]$.

13. Ibid., $\$ 38$, p. 227 [179]. E. Martineau rend Verfallen par échéance.

14. Voir la note de E. Martineau sur la distinction entre échéance et déchéance (p. 318).

15. Ibid., p. $226[178]$. 
rapport dynamique lorsqu'il écrit que “ l'état inauthentique " est « mon état ordinaire tant que je n'ai pas réalisé la conversion à l'authenticité ${ }^{16}$ n.

Sartre discrédite donc les notions d'authenticité et d'inauthenticité, mais conserve néanmoins cette distinction entre deux modes d'être. Héritée de Heidegger, celle-ci est sans doute aussi tributaire de Bergson qui, dans l'Essai sur les données immédiates de la conscience $^{17}$, établit une définition contrastive des deux aspects du moi. Au moi intérieur, profond, à la vie intérieure et individuelle s'opposent le moi extérieur, superficiel, la vie extérieure et sociale. Le second moi recouvre le premier, réel et concret, en est sa représentation symbolique, le "fantôme décoloré ". Pour Bergson, "au sein du moi fondamental, un moi parasite empiétera continuellement sur l'autre ${ }^{18}$ ". L'inauthenticité heideggerienne et le moi social bergsonien se fondent ensemble, chez Sartre, dans la notion de mauvaise foi. Ne pas être vraiment soi-même, c'est fuir ce que l'on est, ne pas se voir tel que l'on est, c'est-à-dire ne pas avoir pris conscience de n'être rien, d'avoir à être, et d'avoir à n’être pas ce quion est.

Si, à la fois pour Bergson, Heidegger et Sartre, l'inauthenticité est une menace inévitable, sinon le mode d'être le plus courant, c'est-àdire que l'authenticité - si tant est qu'on l'ait définie et atteinte -est toujours menacée par l'inauthenticité, ou, comme le dit Sartre, si " la conscience recèle en son être un risque permanent de mauvaisc foi ${ }^{19}$ ", la question qui se pose est celle du passage de l'inauthenticité à l'authenticité. Car, "[s]'il est indifférent d'être de bonne ou do mauvaise foi, parce que la mauvaise foi ressaisit la bonne foi et se glisse à l'origine même de son projet, cela ne veut pas dire qu'on ne puisse échapper radicalement à la mauvaise fo ${ }^{20}$ ". Cette échappée est rendue possible dans $\hat{E}$ tre et temps, de manière toujours provisoire, par l'angoisse, et à travers elle, par le souci, la conscience que l"on prend de sa mort, l'appel à la résolution... C’est cette structure complexe qui peut permettre au Dasein de "secouer le joug du on". c'est-à-dire a aller tout exprès se reprendre, s'extraire de la perte dans le on afin de revenir à lui-même ${ }^{21}$ ". Pour Sartre, si l'on peut dire que la mauvaise foi correspond à l'inauthenticité, c'est aussi l'angoisse -

16. Lêtre et le néant, p. 291.

17. Datant de 1889 , cité à la fois par Heidegger et par Sartre

18. Bergson, Essai sur les données immédiates de la conscience, Paris, PUF, 1927, p. 125.

19. L'être et le néant. p. 107.

20. Ibid., p. 107 , note.

21. Être et temps, $\$ 54$, p. 324 [268]. D'une part, cette reprise du moi authentique s'accomplit pour Bergson pratiquement " dans les circonstances solemnelles " des actions libres (Essai, p. 128). Alors, "le moi d'en bas [...] remonte à la surface ". C'est ce passage à l'authenticité provoqué par un appel qu'expliciteront Les deux sources de la morale et de la religion. D'autre part, théoriquement, la conversion s'opère grâce à une philosophie de l'intuition qui distingue la durée d'un temps qui n'est autre que la spatialisation de cette durée. 
conscience authentique de la liberté ${ }^{22}$ - qui assure la conversion à l'authenticité. Celle-ci est alors la conscience de la liberté absolue du pour-soi, le fait pour la réalité-humaine de prendre conscience de ceci qu'elle ne peut avoir ni remords, ni regret, ni excuse. L'authenticité est ce que Sartre nomme en des termes plus nets et, pour le coup, à la résonance plus morale, quoi qu'il en ait : "une reprise de lềtre pourri par lui-même ${ }^{23}$ ».

Le rapport authenticité-inauthenticité, qui est un problème existentiel de différenciation de modes d'être, est explicité par Sartre, à partir du chapitre III de la deuxième partie de L'être et le néant'. comme une différence entre deux modes de réflexion : la réflcxion pure et la réflexion impure ou complice.

La réflexion pure, simple présence du pour-soi réflexif au pour-soi réfléchii. est à la fois la forme originelle de la réflexion et sa forme idléale ; celle sur le fondenent cle lacpuelle paraît la réflexion impure et celle aussi qui n'est jamais domméc l'abord, celle qu'il faut gagner par une sorte de catharsis. La réflexion impure ou complice [... enveloppe la réflexion pure, mais la dépasse parce qu'clle étend ses prétentions plus loin ${ }^{5}$.

À ce niveau réflexif, le problème du rapport entre une réflexion authentique et une réflexion inauthentique, et du passage entre ces deux réflexions, se formule de la même façon que le problème de la bonne et de la mauvaise foi.

$[\ldots]$ ce qui se donne premièrement dans la vie quoticlienne, c'est la réflexion impure ou constituante, encore qu'elle enveloppe en elle la réflexion pure comme sa structure originelle. Mais celle-ci ne peut être atteinte que par suite cl'unc modification qu'elle opc̀re sur clle-même ct qui est en forme cle catharsis.

$[\ldots]$ la réflexion impure $[\ldots]$ est le mouvement réflexif premier et spontané (mais non originet)

Parce que la conversion de la pensée impure en pensée pure implique une catharsis, la pensée pure est dite aussi "purifiante ". D'un point de vue philosophique, elle est une analyse descriptive, c'est-à-dire qu'elle désigne en fait la méthode phénoménologique elle-même, telle qu'elle fut inaugurée par Husserl, et telle que Heidegger la présente dans le $\$ 7$ de $\hat{E}$ tre et temps. C'est la réflexion complice, ou encore constituante qui est à purifier. En tant que telle, elle est la pensée objectivante qui opère notamment une réification de la conscience sous la forme d'un objet psychique : "c'est la réflexion impure qui constitue la succession des faits psychiques ou

22. L être et le néant, p. 64 ss.

23. Ibid., p. 107, note.

24. Chapitre III. Temporalité originelle et Temporalité psychique : la Réflexion (p. 189 ss).

25. Ibid., p. 194.

26. Ibid., p. 199. Sartre ajoute : "Ce n'est pas le lieu de décrire la motivation et la structure de cette catharsis $n$. 
psyché $^{27}$ ». Elle transforme le pour-soi de la réflexion en en-soi, et se donne comme " un effort avorté du pour-soi pour être autrui en restant $s o i^{28} »$. La réflexion impure peut être dite de mauvaise foi dans la mesure où, par là, elle est " la réflexion qui cherche à déterminer l'être que je suis ${ }^{29}$ ». Être de mauvaise foi, c'est être inauthentique, c'est être sa transcendance " sur le mode de la chose ${ }^{30}$ ". $\hat{\Lambda}$ terme, c'est ronc le sens de la réalité-humaine qui échappe à la réflexion impure - et justement à cause d'elle, en tant qu'elle est un obstacle à la révélation de ce sens. Elle empêche l'accès à la conscience de la liberté, puisque " la réflexion impure et complice |...| appréhende [le manque qu'est le pour-soi| comme objet psychique, c'est-à-dire comme lendance ou comme sentiment "; ce manque - autrement dit, la liberté -. alors «n'est accessible qu'à la réflexion purifiante" ". I'authenticité philosophique est détenue par la phénoménologie. Quant à la complicité de la réflexion impure, elle s'exerce au bénéfice du mode d'être inauthentique, en tentant d'assurer l'hégémonic l'une pensée objectivante inquestionnée ${ }^{32}$.

L'authenticité n'est pas directement prise pour thème. Tout se passe comme si la bonne foi ne pouvait être saisie que négativement par la critique de la mauvaise foi, el que la reflexion pure - pourtant originelle - n"était accessible que par la critique de la raison impure ou complice. Cette critique doit être l'ouvre de la réflexion purifiante. Celle-ci est donc le mouvement de la réflexion pure cherchant à se retrouver elle-même en deçà de la réflexion complice.

Cependant, même si la réflexion purifiante pense ce qu’est la réflexion complice, et même si c'est par elle qu'on peut ainsi distinguer deux modes de réflexion, on constate dans $L$ être et le néant un net déséquilibre entre la réflexion complice et la réflexion purifiante, car la thématisation de la seconde est sans cesse différée. Et, en même temps qu'elle, c'est la prise en compte d'une perspective morale qui, ne trouvant jamais sa place ici, est toujours remise à plus lard. Cöest bien à un au-delà de L'être et le néant quiest promis le léploiement de la réflexion pure-purifiante. En effet, toutes les questions de la fin de la conclusion “ qui nous renvoient à la réflexion pure et non complice, ne peuvent trouver leur réponse que sur le

27. Hbid.. p. 199.

28. Ibid. p. 201.

29. Ibid., p. 210.

30. Ibid., p. 93.

31. Ibid, p. 240

32. Le rapport statique-dynamique entre pensée complice et pensée pure peut être résumé ainsi :

\begin{tabular}{|c|c|}
\hline inauthenticité & authenticité \\
\hline mauvaise foi & bonne foi \\
\hline réflexion impure, complice & réflexion pure, purifiante \\
\hline pensée objectivante & description réflexive \\
\hline
\end{tabular}

<-...-. quotidienneté, chute -...... 
terrain moral ${ }^{33}$ „. Un passage du dernier chapitre de $L$ 'être et le néant est en ceci particulièrement significatif:

Ce type particulier de projet [i.e. le jeu] qui a la liberté pour fondement cr pour but mériterait une étude spéciale. Il se différencie radicalement en effet de tous les autres en ce qu'il vise un type d'être radicalement différent. Il faudrait expliquer tout au long en effet ses rapports avec le projet d'être-Dieu qui nous a paru la structure profonde de la réalité humaine. Mais cette étude ne peut êrre faite ici : elle ressort en effet à une Éthique et elle suppose qu'on ait préalablement défini la nature et le rôle de la réflexion purifiante (nos clescriptions n'ont visé jusqu'ici que la réflexion "complice m) ; elle suppose en outre une prise de position qui ne peut être que morale en face des valeurs qui hantent le Pour-soi ${ }^{31}$.

Dans la mesure où, alors, toute perspective morale s'identifie avec le point de vue de la réflexion purifiante, et oì Sartre, au début de la troisième partie de $L$ 'être et le néant, dit s'appliquer à ne pas a sortir [d'une] attitude de description réflexive ${ }^{35}$, on peut se demander si prendre le parti méthodologique de ne constater que ce qui est, et de ne pas affronter la question de la morale pour elle-même, ne revient pas à assumer en fait le point de vue de la réflexion complice. Ce faisant, ne s'agit-il pas d'une remise en cause. sur le plan pratique, de l'identification de la phénoménologie et de la réflexion pure ${ }^{36}$ ?"

Dans un même mouvement, L'être et le néant débouche sur des perspectives morales, mais se refuse à les suivre. La nécessité d"une: "conversion radicale " est pressentie à plusieurs reprises, mais sa mise en œuvre ne semble pas être du ressort de $I$, être et le néamt:

[Échapper à la mauvaise foi] suppose une reprise de l'être pourri par lui-même (que nous nommerons l'authenticité et dont la description n'a pas sa place ici ${ }^{7}$.

Ces considérations [i.e. sur la haine...] n'excluent pas la possibilité d'une norale de la délivrance et du salut. Mais celle-ci doi être atteinte au terme d'une conversion radicale dont nous ne pouvons parler ici

L'ontologie [...] laisse entrevoir cependant ce que sera une éthique qui prendra ses responsabilités en face d'une réalité humaine en situation ${ }^{*}$.

Dans l'attente de cette morale à venir', l'attitude de la description réflexive ne peut alors qu'offrir une sorte de morale provisoire qui oscille entre le moralisme et un indéterminisme axiologique.

En effet, ce que L'être et le néant donne à entendre, comme Être et temps, c'est un ton tout empreint des accents des moralistes du Grand

33. Ibid., p. 692.

34. Ibid., p. 641,642 .

35. Ibid., p. 265.

36. Ce qui va contre la remarque de Francis Jeanson, qui voit une a identification entre l'attitude phénoménologique et l'attitude éthique * dans Le problème moral et la pensée de Sartre, Paris, Seuil, 1965, p. 241.

37. Ibid., p. 107, note.

38. Ibid., p. 463, note.

39. Ibid., p. 690 . 
Siècle : “ être pourri », “ salut ", " chute », " être-en-faute ${ }^{40}$ „... Certes, il ne faudrait pas être dupe de cette a dureté de l'expression n, contre laquelle Heidegger met en garde au début de $\hat{E}$ tre et temps $(\S 7)^{41}-$ dureté de ton et registre de langue que Sartre accentuera d'ailleurs, dans L'existentialisme est un humanisme, pour qualifier ceux qui se cachent, " par l'esprit de sérieux ou par des excuses déterministes, leur liberté totale", et coux qui essayent de "montrer que leur existence était nécessaire, alors qu'elle est la contingence même de l'apparition de l'homme sur la terre ": les uns sont des "lâches ", les autres des " salauds $»^{42}--$, mais celle-ci incite pourtant à voir comme un saut de la réflexion complice à une réflexion purifiante, qui accentue ce qu'est la réflexion pure. Plus qu'à Kant, c'est à Pascal que l'on pense.

Plus fondamentalement, malgré la définition de la liberté comme source de toute valeur, l'attitude purement descriptive achoppe sur le problème de l'indétermination de la valeur. Sartre, comme IJeidegger, parcourt à rebours le chemin du Tractatus logicophilosophicus de Wittgenstein menant du $\$ 6.4$ " Toutes les propositions sont d'égale valeur" "au $\$ 6.42$ “C'est pourquoi il ne peul pas non plus y avoir de propositions éthiques ${ }^{43}$ ".

Parce que " [l]'ontologie ne saurait formuler elle-même des prescriptions morales", parce qu'elle "s'occupe uniquement de ce qui est, et [qu'] il n’est pas possible de tirer des impératifs de ses indicatifs" "ou, comme le dit Ileidegger, parce que "[d]es consignes " pratiques " $[\ldots \mid$ lappel de la conscience morale n'en donne pas la moindre ${ }^{(1)}{ }_{n}$, L'existentialisme est un humanisme dira qu' " $[\mathrm{o}]$ n peut tout choisir si cest sur le plan de l'engagement libre ${ }^{40} n$ :

[...] toutes les activités humaines sont équivalentes - car elles tendent toutes à sacrificr l'homme pour faire surgir la cause de soi - et [...] toutes sont vouées à

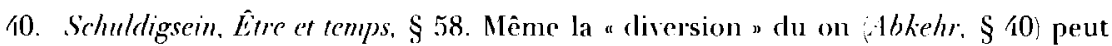
corquer le a divertissement "...

11. Ibid., p. $66|39|$. Eı $\$ 44$, p. 271 [220]: " Le lecours à des preuves de ce type doit éviter de se livrer sans frein à une mystique de mol; pourtant c'est en définitive l'affaire de la philosophie d'empêchei que la force des mots les plus élémentaires où s'exprime le Dasein, ne soit aplatie par le sens commun jusqu'à une inintelligence rui, à son tour, va fonctionner comme une source de faux problèmes ". Néanmoins, on peut être sensible à ce style moraliste, qui se double, dans $L$ être et le néant, d'un style juridique. En effet, en répit de ce qu'affirme Sartre (u cette notion de complicité n'a qu'un sens juridique ", p. 613), cette complicité de la réflexion et la condamnation à la liberté a uous sonmes condamnés à la liberté ", p. 541, par exemple) apportent une nuance judiciaire et pénale aux thèmes de la finitude, du délaissement et de la cléréliction de l'homme.

42. Sartre, L existentialisme est um humanisme, Paris, Nagel, 1970, p. 84, 85.

43. Tractatus, Paris, Gallimard, 1961, p. 103.

44. Lêtre et le néant, p. 690.

45. Être et temps, \$ 59, p. 352 [294].

46. L'existentialisme est un humanisme, p. 88-89. 
l'échec. Ainsi revient-il au même de s'enivrer solitairement ou de conduire les peuples ${ }^{4}$.

La description réflexive ne serait-elle rien d'autre, en fin de compte, que la "complice " de la pensée objective, comme l'affirme Merleau-Ponty ${ }^{48}$ ? Elle l'est, tant qu'elle ne pense pas une liberté réellement pratique et remet à plus tard la question de l'éthique, cl ne peut que le demeurer, tant qu"elle ne sort pas de l'hésitation entre l'assomption d'une éthique négative et le refuge d'une struclure déceptive.

En définitive, cette indétermination pratique est corrélative de l'ajournement de la question de l'éthique, et commune à $\hat{E}$ tre et temps et à $L$ 'être et le néant. De ce fait, la critique sartrienne à l'encontre de $\hat{E}$ tre et temps peut apparaittre comme une autocritique indirecte mais sévère. Et, parce qu’il laisse à maintes reprises en suspens le traitement du problème moral, dont il affirme en même temps la possibilité et la nécessité, L'être et le néant est l'expression d'un manque. Le fondement anhypothétique qu'est la liberté absolue de la réalité-humaine est atteint, mais la question des rapports entre la réflexion pure-purifiante et une raison pratique reste posée. Est-il nécessaire de laisser indéterminée la liberté afin d'en affirmer l'absoluité ? Le refus de toute extériorité doit-il conduire à la liberté d'indifférence? Qu'en est-il, enfin, de la possibilité de penser ensemble une liberté absolue de fait, et des principes qui seraient en droit universalisables? Reconduisant ces questions, L'être et le néant, système délibérément inchoatif, reste une cuvre ouverte.

\section{École Normale Supérieure \\ de Fontenay-St Cloud}

47. L'être et le néant, p. 691.

48. Merleau-Ponty, Phénoménologie de la perception, Paris, Gallimard, 1945, p. 518 : a Notre liberté, dit-on, est ou bien totale, ou bien nulle. Ce dilemme est celui de la pensée objective et de l'analyse réflexive, sa complice ". 